\title{
Comparison of effects of cervical headgear treatment on skeletal facial changes when the treatment time is altered: a randomized controlled trial
}

\author{
Johanna Julku $^{1,2,3}$, Kirsi Pirilä-Parkkinen ${ }^{1,3}$, Mimmi Tolvanen ${ }^{4,5}$, Pertti Pirttiniemii ${ }^{1,2,3}$ \\ ${ }^{1}$ Oral and Maxillofacial Department, Oulu University Hospital ${ }^{2}$ Department of Oral Development \\ and Orthodontics, Research Unit of Oral Health Sciences, University of Oulu ${ }^{3}$ MRC Oulu, Oulu \\ University Hospital and University of Oulu ${ }^{4}$ Department of Community Dentistry, Institute of \\ Dentistry, University of Turku ${ }^{5}$ Center for Life Course Health Research, Faculty of Medicine, \\ University of Oulu, Finland
}

Short title: Dentoskeletal changes, timing and cervical headgear

Correspondence to:

Johanna Julku

Oral and Maxillofacial Department

Oulu University Hospital

PO Box 21

90029 Oys

Finland.

E-mail: johanna.julku@ppshp.fi 


\section{Summary}

Background: There is a lack of evidence based on longitudinal information in the field of Class II malocclusion management with cervical headgear $(\mathrm{CH})$, especially in a randomized setting.

Objectives: The main objective of this study was to evaluate skeletal facial changes, particularly in vertical dimensions, after Kloehn-type $\mathrm{CH}$ treatment in children when the timing of treatment is altered.

Trial design: Prospective, parallel-group, randomized controlled trial.

Methods: Screened children with Class II malocclusion were randomized in 1:1 ratio to two groups of equal size by sealed-envelope randomization: the early group (EG), where active $\mathrm{CH}$ treatment was started at the age of 7.8 years, and the late group (LG), where $\mathrm{CH}$ treatment was started at the age of 9.5 years. The active treatment was continued until normal Class I occlusion on first molars was achieved. Cephalograms were taken at three different time points. Changes in cephalometric measurements were compared between groups and genders. Blinding was applicable for outcome evaluation.

Results: Of 67 randomized children, 56 completed the study. Upper facial height increased during the $\mathrm{CH}$ treatment phase, as the parameter $\mathrm{N}-\mathrm{ANS}$ increased significantly during the active treatments of EG $(P<0.05)$ and LG $(P<0.05)$. Also, the parameter NSL-PL increased significantly during the treatment of EG $(P<0.01)$ and during the treatment of $\operatorname{LG}(P<0.01)$. The Gonial angle decreased significantly in the early $\mathrm{CH}$ treatment group compared to the later treatment group $\left(\mathrm{T}_{0}-\mathrm{T}_{2}: P<0.01\right)$. $\mathrm{CH}$ improved the anteroposterior jaw relationship. No harms were encountered.

Conclusions: Although the upper facial height increased, the mandible showed anterior rotation after $\mathrm{CH}$ treatment. The Gonial angle was significantly decreased in the EG compared to the LG. There were gender-specific differences in both sagittal and vertical dimensions when examining interrelations in dimensional changes. The differences found between the early and later treatment groups were not clinically important when the cephalometric results are considered.

Clinical Registration: ClinicalTrials.gov (NCT02010346).

Keywords: Class II malocclusion; Cervical headgear; Timing; Randomized controlled trial 


\section{Introduction}

Since its introduction, headgear has been widely used in treatment of Class II malocclusion, and early work with headgear laid the foundation for the use of the cervical headgear $(\mathrm{CH})$ known today $(1,2)$. Since then, the effects of $\mathrm{CH}$ on craniofacial structures have raised many questions, despite extensive research on the subject. The most well-known findings of these studies are the effects on the maxilla. The $\mathrm{CH}$ inhibits forward growth of the maxilla, the maxilla moves distally, and simultaneously, the palatal plane rotates downwards anteriorly (3-8). On the other hand, these changes have been shown to be reversible in long-term follow-up $(7,9)$.

Distal movement and a slight extrusion of maxillary first molars have also been reported (4-6, 10). This change in the position of the upper molars has been suggested to be responsible for a posterior rotation of the mandible $(5,6)$. For this reason, the use of $\mathrm{CH}$ is not recommended in patients with vertical growth pattern, even though there are reports where the use of $\mathrm{CH}$ with vertical growth pattern did not worsen the posterior growth (11). On the contrary, anterior rotation of the mandible has been reported $(11,12)$.

Most studies of $\mathrm{CH}$ treatment have concluded this rotation of the mandible to be a secondary effect, and there are only few studies of the detailed effects of $\mathrm{CH}$ on mandibular growth in sagittal and vertical dimensions. These studies have shown that $\mathrm{CH}$ treatment does not alter the mandibular plane angle and that vertical dimensions of the face are generally not affected (13-15). It has been stated that after $\mathrm{CH}$ treatment, the mandible grows forward, following the normal individual growth pattern, and that treatment does not have adverse effects on vertical growth $(4,7,16,17)$.

Gender distribution is usually described in study materials, but its effect on treatment outcomes has generally been disregarded. There are a few reported differences between genders after $\mathrm{CH}$ treatment, but these are mostly considered to be gender-specific growth changes and not caused by $\mathrm{CH}$ treatment $(4,13)$. Recent evidence has shown that when the timing of the treatment is altered, gender does have an effect on treatment outcomes $(8,18)$.

Although $\mathrm{CH}$ has been widely used and extensively studied, systematic reviews and meta-analyses have revealed that there are only a few prospective randomized clinical trials of the effects of $\mathrm{CH}$ treatment on Class II malocclusion. These reports commonly suffer from lack of a proper control group or have been based on retrospective material. Some studies have looked at the dentofacial 
effects of $\mathrm{CH}$ compared to other orthodontic devices (19-22). For this reason, it is important to examine these longitudinal changes in detail in a randomized setting.

\section{Objective}

The aim of this study was to evaluate skeletal facial changes, especially in vertical dimensions, after Kloehn-type $\mathrm{CH}$ treatment in children when timing of treatment is altered. The hypothesis was that the timing of treatment affects the growth changes in the examined structures.

\section{Subjects and Methods}

\section{Study Design, registration and ethical issues}

This study is a prospective, parallel-group, controlled trial randomized in 1:1 ratio and reported according to the CONSORT 2010 statement guidelines (23). The trial is registered at ClinicalTrials.gov (NCT02010346), and before start, the study protocol was approved by the Ethics Committee of the Oulu University Hospital, Finland (EETTMK: 46/2003) and by the health service authorities in the municipalities involved in the study.

\section{Sample size calculation}

The sample size was calculated with independent samples $t$-test at a significance level of 0.05 and a power of 80 per cent, resulting in a sample size of eleven in each group (7). Our study is a longitudinal follow-up study where the patients are followed from ages 7 to 18 years. Because long studies are prone to dropouts, to secure the completion of the study the intake was increased. Also, if the results would show a reason why it is necessary to do split analyses, for example, if the gender is reflected to the results, the size of the subgroups would be sufficient.

\section{Participants and eligibility criteria}

The patients were selected from birth cohorts in three municipalities in northern Finland between February 2004 and June 2008, and 7-year-old schoolchildren were screened for the study by clinicians in health centres. The inclusion criteria were Class II occlusion and overjet $6 \mathrm{~mm}$ or more and deep bite. The exclusion criteria were previous orthodontic treatment and PL-ML angle (the angle between the palatal line and the mandibular line) over 35 degrees. In addition, children with inborn facial syndrome and severe facial asymmetry were not included. Sixty-seven children with Class II malocclusion (mean age 7.2 years, standard deviation (SD) 0.55, 28 females, 39 males) met the inclusion criteria and were included in the study and randomized into two groups. 
The sample size calculation, screening and randomization as well as blinding have been described in detail in previous articles of this study $(8,18)$.

\section{Interventions}

The children were randomly divided into two equal-sized groups: the early group (EG) and the late group (LG). A treatment protocol with $\mathrm{CH}$ treatment was carried out for all children. In the EG ( $n=$ 33; 13 female and 20 male), $\mathrm{CH}$ treatment was started after eruption of the first maxillary molars (mean age 7.8 years, SD: 0.53) and continued until normal Class I occlusion on molars was achieved. Reduced use of $\mathrm{CH}$ was applied after active treatment when necessary. In the LG ( $n=34$; 15 female and 19 male), equivalent $\mathrm{CH}$ therapy was performed proximately one and half years later (mean age 9.5 years, SD: 0.59).

A Kloehn-type $\mathrm{CH}$ with a long outer bow and maxillary first molar bands with gingival tubes was used. The outer bows were bent 10 degrees upwards and the inner bow was held $5 \mathrm{~mm}$ wider than the distance between the gingival tubes. An orthodontic force of $500 \mathrm{~g}$ was provided. The duration of $\mathrm{CH}$ use was $8-10$ hours during the night. The $\mathrm{CH}$ therapy was carried out by clinicians in community health centres. A more detailed description of the interventions is available in the previous articles $(8,18)$.

\section{Participant flow}

Patient flow through the study is illustrated in Figure 1. A total of 270 children were assessed for eligibility. 67 subjects were enrolled in the study and randomized to either early or later treatment groups. The primary analysis $\left(\mathrm{T}_{0}\right)$ was intended for all randomly assigned patients. During the first follow-up, 3 patients in the EG and 2 in the LG dropped out from the study. 62 children participated in the second analysis $\left(\mathrm{T}_{1}\right)$. During the second follow-up, 4 patients in the EG and 2 in the LG dropped out. A total of 56 children completed the second follow-up $\left(\mathrm{T}_{2}\right)$. A more detailed description of the participants is available in the previous articles $(8,18)$. Of the 67 subjects at the baseline, 6 subjects did not attend in either analysis $\mathrm{T}_{1}$ or $\mathrm{T}_{2}$, but they did not differ from those who did, in terms of group or gender, or any of the linear or angular measurements at $\mathrm{T}_{0}$.

\section{Outcomes}


Lateral cephalograms were taken from all the subjects at three different time points during the follow-up. The cephalograms were taken at baseline ( $\mathrm{T}_{0}$, mean age 7.3 years, $\mathrm{SD}$ : 0.53$)$, at the beginning of treatment in the LG ( $\mathrm{T}_{1}$, mean age 9.6 years, SD: 0.51$)$, and at the end of treatment in the $L G\left(T_{2}\right.$, mean age 11.5 years, SD: 0.57$)$.

Cephalometric software (WinCeph 8.0, Rise Corporation, Japan) was used for cephalometric evaluation. Lateral cephalograms were calibrated in regard to magnification before the landmark definition. The evaluation and landmark digitalization was carried out by one of the authors (JJ). Cephalograms with poor quality for measuring were excluded. The cephalometric points, reference lines, and explanation of the less used cephalometric measurements used in the analysis are shown in Figures 2 and 3.

\section{Statistical analysis}

Statistical significances for difference between the groups at each time point were evaluated using Mann-Whitney $U$-test, and for difference between the groups in change during study periods using general linear model for repeated measures. In the mean values of each time point, all participants at that time point were included. In mean change scores, only those who had cephalograms for both time points were included. To evaluate whether the changes in SNA and facial axis $\left(\mathrm{T}_{0}-\mathrm{T}_{2}\right)$ were associated with changes in other measures, Pearson's correlation coefficients between change scores were calculated. To evaluate whether the baseline measure of facial axis was associated with changes in other measures during the study, correlation coefficients between facial axis $\mathrm{T}_{0}$ and change scores $\mathrm{T}_{0}-\mathrm{T}_{2}$ were used.

\section{Examiner reliability}

To determine the error of the method, 20 randomly selected lateral cephalograms were traced and measured twice with a 2 -week interval. The intra-rater reliability was measured by intraclass correlation coefficient (ICC) to compare the repeated measurements. ICCs for linear measures were good/excellent (range: 0.807-0.992) for other measures but moderate for U6(d)-PtV (0.561). In angular measures, ICCs were good/excellent (0.828-0.969) for other measures but moderate for NSL-PL (0.692).

\section{Results}

\section{Outcomes}




\section{Cephalometric measurements between the groups (genders combined)}

Table 1 shows the linear measurements at different time points and changes during the study periods. N-ANS changed differently among the groups; the increase was significantly higher in EG during $\mathrm{T}_{0}-\mathrm{T}_{1}$, and in $\mathrm{LG}$ during $\mathrm{T}_{1}-\mathrm{T}_{2}$. At $\mathrm{T}_{2}$, the value of $\mathrm{N}-\mathrm{ANS}$ was at the same level in both groups. Convexity $(\mathrm{mm})$ decreased in both groups, but at different time periods: during $\mathrm{T}_{0}-\mathrm{T}_{1}$ in EG and during $\mathrm{T}_{1}-\mathrm{T}_{2}$ in $\mathrm{LG}$. A decrease in $\mathrm{U6}(\mathrm{d})-\mathrm{PtV}$ value was seen in EG during $\mathrm{T}_{0}-\mathrm{T}_{1}$ compared to LG.

Changes in angular measurements at different time points and study periods are shown in Table 2. Differences in changes in the angles SNA, ANB and angle of convexity were seen between the groups at both time periods. In the EG, decreases occurred during the first time period and in the LG, during the second time period. An increase in NSL-PL angle was also seen in EG at $\mathrm{T}_{0}-\mathrm{T}_{1}$ and in $L G$ at $\mathrm{T}_{1}-\mathrm{T}_{2}$. During the study, Gonial angle decreased significantly more among EG than among $\mathrm{LG}$ at $\mathrm{T}_{0}-\mathrm{T}_{2}$. There was a significant difference in $\mathrm{OL}-\mathrm{ML}$ angle at $\mathrm{T}_{1}-\mathrm{T}_{2}$ between the groups. During $\mathrm{T}_{1}-\mathrm{T}_{2}$, OL-ML angle decreased among EG but increased among LG. The linear and angular measurements during the follow-ups and at the end of the follow-up did not reveal any gender differences between the results.

\section{Correlations between changes in SNA angle and cephalometric measurements (genders separated)}

Table 3 shows the correlation of change in SNA angle at $\mathrm{T}_{0}-\mathrm{T}_{2}$ to changes in linear measurements at $\mathrm{T}_{0}-\mathrm{T}_{2}$. The change in SNA correlated differently to linear changes between genders. Significant correlations were seen in EG males and LG females, but they were opposite. Negative correlations were seen in parameters $\mathrm{N}-\mathrm{ANS}, \mathrm{N}-\mathrm{Me}, \mathrm{Cd}-\mathrm{A}, \mathrm{Cd}-\mathrm{Gn}$ and $\mathrm{Cd}-\mathrm{Go}$ among EG males. In LG females, the correlation was opposite and positive correlations were seen in parameters ANS-Me, $\mathrm{Cd}-\mathrm{A}, \mathrm{Cd}-\mathrm{Gn}, \mathrm{S}-\mathrm{tGo}$ and convexity $(\mathrm{mm})$. The change in SNA angle was negatively correlated with the change in N-ANS and positively correlated with the change in U6(d)-PtV among EG females.

Table 4 shows the correlation of change in SNA angle at $\mathrm{T}_{0}-\mathrm{T} 2$ to changes in angular measurements at $\mathrm{T}_{0}-\mathrm{T}_{2}$. The change in SNA was positively correlated with the changes in sagittal angles SNB and facial plane angle among both groups and genders. No other correlations were observed among EG males. Positive correlations were observed in the change of SNA with the changes in sagittal parameters ANB and angle of convexity among LG females and in the change of 
vertical parameter facial axis among EG females and LG males. Negative correlations in the change of SNA were observed with the changes of vertical parameters NSL-PL among EG females and LG males, and NSL-ML among LG females and males. Negative correlations were seen between the change in SNA and angles NSL-RL and NSL-OL among both genders of LG.

\section{Correlations between changes in facial axis and cephalometric measurements (genders separated)}

Table 5 shows the correlation of change in facial axis at $\mathrm{T}_{0}-\mathrm{T}_{2}$ to changes in angular measurements at $\mathrm{T}_{0}-\mathrm{T}_{2}$. The change in facial axis was positively correlated with the change in facial plane angle among both groups and genders. Positive correlations were observed between the change of facial axis and change of sagittal parameters SNA among EG females and LG males and SNB among EG females and males and LG males. In vertical parameters, a positive correlation was observed between the change of facial axis and change of Gonial angle among LG females. The change in facial axis was negatively correlated with the change in NSL-RL among both groups and genders. The change in facial axis correlated negatively with vertical parameters NSL-PL among EG females, with NSL-ML among EG females and LG males and with NSL-OL among EG males and LG males.

The correlations between facial axis at $T_{0}$ and angular measurement changes in $T_{0}-T_{2}$ were seen only in LG males. Sagittal parameters SNA, SNB, and facial plane angle correlated negatively with the facial axis at $\mathrm{T}_{0}$; on the contrary, vertical parameters NSL-ML, NSL-RL, and NSL-OL correlated positively with facial axis. The facial axis at $\mathrm{T}_{0}$ correlated negatively with the change of facial axis in $\mathrm{T}_{0}-\mathrm{T}_{2}$ among $\mathrm{LG}$ males.

\section{Harms}

No harms were encountered during the follow-up between $\mathrm{T}_{0}-\mathrm{T}_{2}$.

\section{Discussion}

\section{Interpretation}

Although Class II malocclusion and its treatments have been widely studied, there is a lack of clinical trials providing high-quality evidence-based information on the management of Class II malocclusion and the true effects of $\mathrm{CH}$ treatment on the craniofacial complex $(21,24)$. The complexity of the Class II malocclusion, the variations in headgear design and the use of 
retrospective material in numerous studies have given significant amount of information, but this information has also been shown to be inconsistent.

This randomized controlled trial was aimed to evaluate skeletal facial changes in children after early or later timed $\mathrm{CH}$ treatment. We have earlier shown the effects of treatment timing and gender on the upper airway and related skeletal structures in $\mathrm{CH}$ treatment (8). Here, the genders were first pooled together to study further the effect of timing especially on the skeletal vertical parameters. The genders were, however, examined separately to examine the longitudinal interrelations of facial structures.

The idea was to study especially timing of the early treatment (EG) during the early mixed dentition compared to the later treatment (LG) starting around the onset of late mixed dentition. The outcome of this study could be different if the groups were treated in different growth stages. However, it is noteworthy that in many studies on $\mathrm{CH}$ treatment, the initiation of treatment has been done between 8.5 and 10.5 years, before or during the initiation of the second mixed dentition $(4,5,11,12)$.

The results of this study show that the $\mathrm{CH}$ treatment improves the antero-posterior relationship of the maxilla and the mandible. The decrease in the convexity of the middle face and in the SNA angle shows the restriction of maxillary forward growth, especially during early treatment, which is in accordance with the previously reported findings $(8,25-27)$. However, due to normal growth, the midfacial length increased despite the restricting force of $\mathrm{CH}$ on the maxilla. Our results also showed normal growth of the mandible. This is in concordance with previous studies in children with normal occlusion $(28,29)$. It has also been shown that dentofacial growth of untreated Class II children does not notably differ from growth of children with normal occlusion (30). The results indicated that the most noticeable increase in mandibular length occurred during early treatment phase $\left(\mathrm{T}_{0}-\mathrm{T}_{1}\right)$ in both groups regardless of the treatment of the EG. This partly explains the decrease in the angle of convexity and improvement of the antero-posterior jaw relationship, which were more prominent in the early-treated children.

Our results showed slight distalization of the upper first molars after early $\mathrm{CH}$ treatment. At the end of the second follow-up, the anterior growth of the maxilla overcomes the distal movement of upper molars. Our previous study of dental arch effects after $\mathrm{CH}$ showed that the movement of upper molars is seen in significant lengthening of the dental arch, pointing out that the movement is mostly dental in nature (18). 
Downward rotation of the palatal plane anteriorly and increase in the upper anterior face height was seen in both EG and LG immediately after active $\mathrm{CH}$ treatment. This supports the previous reports on $\mathrm{CH}$ treatment $(4,6,8,31)$. The force vector created by $\mathrm{CH}$ works below the centre of resistance of the maxilla, changing the growth direction and causing the palatal plane to rotate downward anteriorly. It is interesting that the timing of treatment did not affect the magnitude of change.

The decrease in the mandibular plane angle showed anterior rotation of the mandible in both treatment groups. Our finding of the anterior rotation of the mandible is in concordance with previous studies $(4,11,12)$. Several studies have reported an opposite result, a posterior rotation of the mandible after $\mathrm{CH}$ treatment $(5,6,32)$. This has led to the assumption that $\mathrm{CH}$ has a tendency to open the bite, especially in patients with vertical growth pattern. This has in turn resulted in avoiding the use of $\mathrm{CH}$ to prevent undesirable side-effects, such as posterior rotation of the mandible and an increase in lower anterior face height. Studies looking the effect of $\mathrm{CH}$ treatment on patients with different mandibular plane angles and growth patterns have shown that mandibular rotation responses differ with low and high angle groups $(33,34)$. It has been stated that there is less anterior mandibular rotation seen after $\mathrm{CH}$ treatment with the high angle group (34). On the other hand, it has also been found that patients with pronounced horizontal growth show bite opening after $\mathrm{CH}$ treatment $(11,33)$. These might be the possible causes to different outcomes of the $\mathrm{CH}$. It has to take into account that in our study children with PL-ML angle over 35 degrees were excluded. Thus the most severe high angle and low angle patients were not present at the groups and this might reflect to the results.

A longitudinal growth study with untreated Class II subjects has shown that the Gonial angle decreases during normal growth (35). According to this study, the annual change in Gonial angle was less than 1 degree. A previous study of the effects $\mathrm{CH}$ treatment on growth patterns has shown that $\mathrm{CH}$ reduces the mandibular angle (ArGoMe) (11). This study shows a similar effect and a decrease in the Gonial angle in both treatment groups during $\mathrm{T}_{0}-\mathrm{T}_{2}$ after active treatment. Most of the changes occurred during the first follow-up $\left(\mathrm{T}_{0}-\mathrm{T}_{1}\right)$ and the decrease in the Gonial angle after early $\mathrm{CH}$ treatment was significant compared to later treatment. This is probably a consequence of the prepubertal growth spurt. A study with Class II patients has shown that children with a Gonial angle less than 125.5 degrees are favourable candidates for future functional jaw orthopaedics (FJO) (36). This study showed that $\mathrm{CH}$ treatment significantly decreases the Gonial angle, and it could be 
speculated that especially early $\mathrm{CH}$ treatment improves the Class II patients' response to possible forthcoming FJO by decreasing the Gonial angle.

There was difference between the genders in how the decrease in the SNA angle $\left(\mathrm{T}_{0}-\mathrm{T}_{2}\right)$ correlated to other cephalometric changes. Significant correlations in linear measurements were seen in EG males and LG females, but they were opposite in direction. In those EG males whom SNA decreased the most, both the vertical and sagittal parameters showed the largest increase. In contrast, in LG females the correlation was opposite, with measurements decreasing alongside with SNA. In angular measurements, correlations with the change in the SNA angle were seen among both groups and genders, but there were differences between the sagittal and the vertical changes. The change in SNA correlated positively with the sagittal measurement changes and negatively with the vertical changes, indicating a decrease in sagittal and an increase in vertical parameters.

Between the correlations of the change in facial axis and the angular measurement changes at $\mathrm{T}_{0}-$ $\mathrm{T}_{2}$, an outcome analogous to the change in SNA angle correlations was seen. Correlations were observed among both groups and genders, positive correlations were seen in the change in sagittal parameters, whereas negative correlations were seen in the vertical parameters. Correlations between facial axis at $T_{0}$ and angular measurement changes in $\mathrm{T}_{0}-\mathrm{T}_{2}$ were seen only in $\mathrm{LG}$ males. Sagittal parameters decreased the most among those with the largest facial axis at $\mathrm{T}_{0}$, whereas on the contrary, vertical parameters increased the most. In those LG males who had the largest facial axis at $\mathrm{T}_{0}$, the facial axis showed the largest decrease.

These findings suggest that the more the $\mathrm{CH}$ restricts the forward growth of the maxilla, the more the vertical portions of the face increase, pointing to opening rotation in angular parameters. An increase in linear measurements, especially in EG males, suggests that normal growth overcomes the opening rotation and normal growth rotation occurs. At the end of the follow-up, the vertical and horizontal changes in facial dimension linear measurements did not show significant differences between the groups. It is important that children with remarkable skeletal bite opening were excluded from the study. They might respond differently to $\mathrm{CH}$ treatment than children with normal growth pattern in the change of facial axis. Most of the changes in dimensions occurred during the first follow-up $\left(\mathrm{T}_{0}-\mathrm{T}_{1}\right)$. The linear and angular measurements during the follow-ups and at the end of the follow-up did not reveal any gender differences in results.

\section{Limitations}


Lack of a proper control group could be seen as weakness of this study. However, it is not ethical to use untreated Class II malocclusion patients for long-term controls, and the use of historical control groups has been shown to weaken the treatment effects (37). As the timing of the treatment was the subject to be investigated, the two treatment groups served as controls for one another. The differences between normal growth and treatment effect were shown during the treatment of the EG and the follow-up of the $\mathrm{LG}$ at $\mathrm{T}_{0}-\mathrm{T}_{1}$.

The ICC for one linear measurement and one angular measurement was considered moderate, but these two results were still reported. The effect of these measurements should be interpreted with discretion.

Because the aim was to study long-term effects of $\mathrm{CH}$ treatment and there were no data from dropouts in later time points, per-protocol analyses were used, and in every step, data were used from all subjects that it was available. Intention-to-treat analysis is recommended in the CONSORT 2010 statement guidelines, but our data were not suitable for it (23). As a result, there might be a post-randomization bias and false-positive results (38). However, there were only six participants who attended only at baseline, and based on the dropout analysis, they did not differ from those who attended also later.

\section{Generalization}

The result of this study can be generalized to children with Class II malocclusion. On the basis of these results, $\mathrm{CH}$ is a competent treatment option in both early and later treatment phases.

\section{Conclusion}

Although the upper facial height increased during active $\mathrm{CH}$ treatment in both groups, an anterior rotation of the mandible was seen in both treatment groups after $\mathrm{CH}$ treatment. This is likely due to remodelling in the gonial area. At the end of the follow-up, there were no major differences in linear and angular measurements caused by the effects of the timing of treatment or gender. There was a gender difference in the response to treatment with $\mathrm{CH}$ in inter-correlations of the cephalometric values. The differences between the early and later treatment groups were not clinically important when the cephalometric results are considered.

\section{Protocol}


The protocol was not published prior to trial commencement.

\section{Funding}

This work was supported by the Kerttu Saalasti Foundation.

\section{Acknowledgements}

The authors gratefully acknowledge the practitioners at the health centres of Oulunsalo, Kemi and Haukipudas and the children and their parents for participating in the study. We would like to thank Mr Reijo Kettunen and Ms Seija Leskelä for their valuable work with the figures.

\section{Conflict of interest}

None to declare.

\section{References}

1. Kloehn, S.J. (1947) Guiding alveolar growth and eruption of teeth to reduce treatment time and produce a more balanced denture and face. The Angle Orthodontist, 17, 10-33.

2. Kloehn, S.J. (1953) Orthodontics - force or persuasion. The Angle Orthodontist, 23, 56-65.

3. Baumrind, S., Korn, E.L., Isaacson, R.J., West, E.E. and Molthen, R. (1983) Quantitative analysis of the orthodontic and orthopedic effects of maxillary traction. American Journal of Orthodontics, 84, 384-398.

4. Kirjavainen, M., Kirjavainen, T., Hurmerinta, K. and Haavikko, K. (2000) Orthopedic cervical headgear with an expanded inner bow in Class II correction. The Angle Orthodontist, 70, 317-325.

5. Melsen, B. (1978) Effects of cervical anchorage during and after treatment: an implant study. American Journal of Orthodontics, 73, 526-540.

6. Wieslander, L. (1974) The effect of force on craniofacial development. American Journal of Orthodontics, 65, 531-538.

7. Mäntysaari, R., Kantomaa, T., Pirttiniemi, P. and Pykäläinen, A. (2004) The effects of early headgear treatment on dental arches and craniofacial morphology: a report of a 2 year randomized study. European Journal of Orthodontics, 26, 59-64.

8. Julku, J., Pirilä-Parkkinen, K. and Pirttiniemi, P. (2018) Airway and hard tissue dimensions in children treated with early and later timed cervical headgear - a randomized controlled trial.

European Journal of Orthodontics, 40, 285-295. 
9. Pirttiniemi, P., Kantomaa, T., Mäntysaari, R., Pykäläinen, A., Krusinskiene, V., Laitala, T. and Karikko, J. (2005) The effects of early headgear treatment on dental arches and craniofacial morphology: an 8 year report of a randomized study. European Journal of Orthodontics, 27, 429436.

10. Kang, S.J., Kim, H.H., Hwang, H.S. and Lee, K.M. (2017) Immediate changes in the mandibular dentition after maxillary molar distalization using headgear. The Korean Journal of Orthodontics, 47, 142-147.

11. Godt, A., Kalwitzki, M. and Göz, G. (2007) Cervical headgear treatment and growth patterns: analysis by lateral cephalometry. Journal of Orofacial Orthopedics, 68, 38-46.

12. Sambataro, S., Fastuca, R., Oppermann, N.J., Lorusso, P., Baccetti, T., Franchi, L. and Caprioglio, A. (2017) Cephalometric changes in growing patients with increased vertical dimension treated with cervical headgear. Journal of Orofacial Orthopedics, 78, 312-320.

13. Hubbard, G.W., Nanda, R.S. and Currier, G.F. (1994) A cephalometric evaluation of nonextraction cervical headgear treatment in Class II malocclusions. The Angle Orthodontist, 64, 359-370.

14. Lima Filho, R.M.A. and de Oliveira Ruellas, A.C. (2007) Mandibular behavior with slow and rapid maxillary expansion in skeletal Class II patients: a long-term study. The Angle Orthodontist, $77,625-631$.

15. Lione, R., Franchi, L., Laganà, G. and Cozza, P. (2015) Effects of cervical headgear and pendulum appliance on vertical dimension in growing subjects: a retrospective controlled clinical trial. European Journal of Orthodontics, 37, 338-344.

16. Lima Filho, R.M.A., Lima, A.L. and de Oliveira Ruellas, A.C. (2003) Mandibular changes in skeletal Class II patients treated with Kloehn cervical headgear. American Journal of Orthodontics and Dentofacial Orthopedics, 124, 83-90.

17. Zervas, E.D., Galang-Boquiren, M.T.S., Obrez, A., Costa Viana, M.G., Oppermann, N., Sanchez, F., Romero, E.G. and Kusnoto, B. (2016) Change in the vertical dimension of Class II Division 1 patients after use of cervical or high-pull headgear. American Journal of Orthodontics and Dentofacial Orthopedics, 150, 771-781.

18. Julku, J., Hannula, M., Pirilä-Parkkinen, K., Tolvanen, M. and Pirttiniemi, P. (2019) Dental arch effects after early and later timed cervical headgear treatment - a randomized controlled trial. European Journal of Orthodontics, in press.

19. Antonarakis, G.S. and Kiliaridis, S. (2007) Short-term anteroposterior treatment effects of functional appliances and extraoral traction on Class II malocclusion: a meta-analysis. The Angle Orthodontist, 77, 907-914.

20. Henriques, F.P., Janson, G., Henriques, J.F.C. and Pupulim, D.C. (2015) Effects of cervical headgear appliance: a systematic review. Dental Press Journal of Orthodontics, 20, 76-81.

21. Nucera, R., Militi, A., Lo Giudice, A., Longo, V., Fastuca, R., Caprioglio, A., Cordasco, G. and Papadopoulos, M.A. (2018) Skeletal and dental effectiveness of treatment of Class II malocclusion 
with headgear: a systematic review and meta-analysis. The Journal of Evidence-Based Dental Practice, 18, 41-58.

22. Papageorgiou, S.N., Kutschera, E., Memmert, S., Gölz, L., Jäger, A., Bourauel, C. and Eliades, T. (2017) Effectiveness of early orthopaedic treatment with headgear: a systematic review and meta-analysis. European Journal of Orthodontics, 39, 176-187.

23. Schulz, K.F., Altman, D.G. and Moher, D. (2010) CONSORT 2010 Statement: updated guidelines for reporting parallel group randomised trials. British Medical Journal, 340, 698-702.

24. Millett, D.T., Cunningham, S.J., O'Brien, K.D., Benson, P.E. and de Oliveira, C.M. (2012) Treatment and stability of Class II Division 2 malocclusion in children and adolescents: a systematic review. American Journal of Orthodontics and Dentofacial Orthopedics, 142, 159-169.

25. Lima Filho, R.M.A. and Ruellas, A.C.O. (2007) Long-term anteroposterior and vertical maxillary changes in skeletal Class II patients treated with slow and rapid maxillary expansion. The Angle Orthodontist, 77, 870-874.

26. Siqueira, D.F., de Almeira, R.R., Janson, G., Brandão, A.G. and Coelho Filho, C.M. (2007) Dentoskeletal and soft-tissue changes with cervical headgear and mandibular protraction appliance therapy in the treatment of Class II malocclusions. American Journal of Orthodontics and Dentofacial Orthopedics, 131, 447.e21-447.e30.

27. Mossaz, C.F., Byloff, F.K. and Kiliaridis, S. (2007) Cervical headgear vs pendulum appliance for the treatment of moderate skeletal Class II malocclusion. American Journal of Orthodontics and Dentofacial Orthopedics, 132, 616-623.

28. Baccetti, T., Franchi, L., McNamara Jr., J.A. and Tollaro, I. (1997) Early dentofacial features of Class II malocclusion: a longitudinal study from the deciduous through the mixed dentition. American Journal of Orthodontics and Dentofacial Orthopedics, 111, 502-509.

29. Vásquez, M.J., Baccetti, T., Franchi, L. and McNamara Jr., J.A. (2009) Dentofacial features of Class II malocclusion associated with maxillary skeletal protrusion: a longitudinal study at the circumpubertal growth period. American Journal of Orthodontics and Dentofacial Orthopedics, 135, 568.e1-568.e7.

30. Baccetti, T., Stahl, F. and McNamara Jr., J.A. (2009) Dentofacial growth changes in subjects with untreated Class II malocclusion from late puberty through young adulthood. American Journal of Orthodontics and Dentofacial Orthopedics, 135, 148-154.

31. Ülger, G., Arun, T., Sayinsu, K. and Isik, F. (2006) The role of cervical headgear and lower utility arch in the control of the vertical dimension. American Journal of Orthodontics and Dentofacial Orthopedics, 130, 492-501.

32. Baumrind, S., Molthen, R., West, E.E. and Miller, D.M. (1978) Mandibular plane changes during maxillary retraction. American Journal of Orthodontics, 74, 32-40.

33. Godt, A., Kalwitzki, M. and Göz, G. (2007) Effects of cervical headgear on overbite against the background of existing growth patterns: a retrospective analysis of study casts. The Angle Orthodontist, 77, 42-46. 
34. Haralabakis, N.B. and Sifakakis, I.B. (2004) The effect of cervical headgear on patients with high or low mandibular plane angles and the "myth" of posterior mandibular rotation. American Journal of Orthodontics and Dentofacial Orthopedics, 126, 310-317.

35. Stahl, F., Baccetti, T., Franchi, L. and McNamara Jr., J.A. (2008) Longitudinal growth changes in untreated subjects with Class II Division 1 malocclusion. American Journal of Orthodontics and Dentofacial Orthopedics, 134, 125-137.

36. Franchi, L. and Baccetti, T. (2006) Prediction of individual mandibular changes induced by functional jaw orthopedics followed by fixed appliances in Class II patients. The Angle Orthodontist, 76, 950-954.

37. Papageorgiou, S.N., Koretsi, V. and Jäger, A. (2017) Bias from historical control groups used in orthodontic research: a metaepidemiological study. European Journal of Orthodontics, 39, 98-105.

38. Bondemark, L. and Abdulraheem, S. (2018) Intention to treat (ITT) analysis as reported in orthodontic randomized controlled trials - evaluations of methodology and recommendations for the accurate use of ITT analysis and handling dropouts. European Journal of Orthodontics, 40, 409413. 


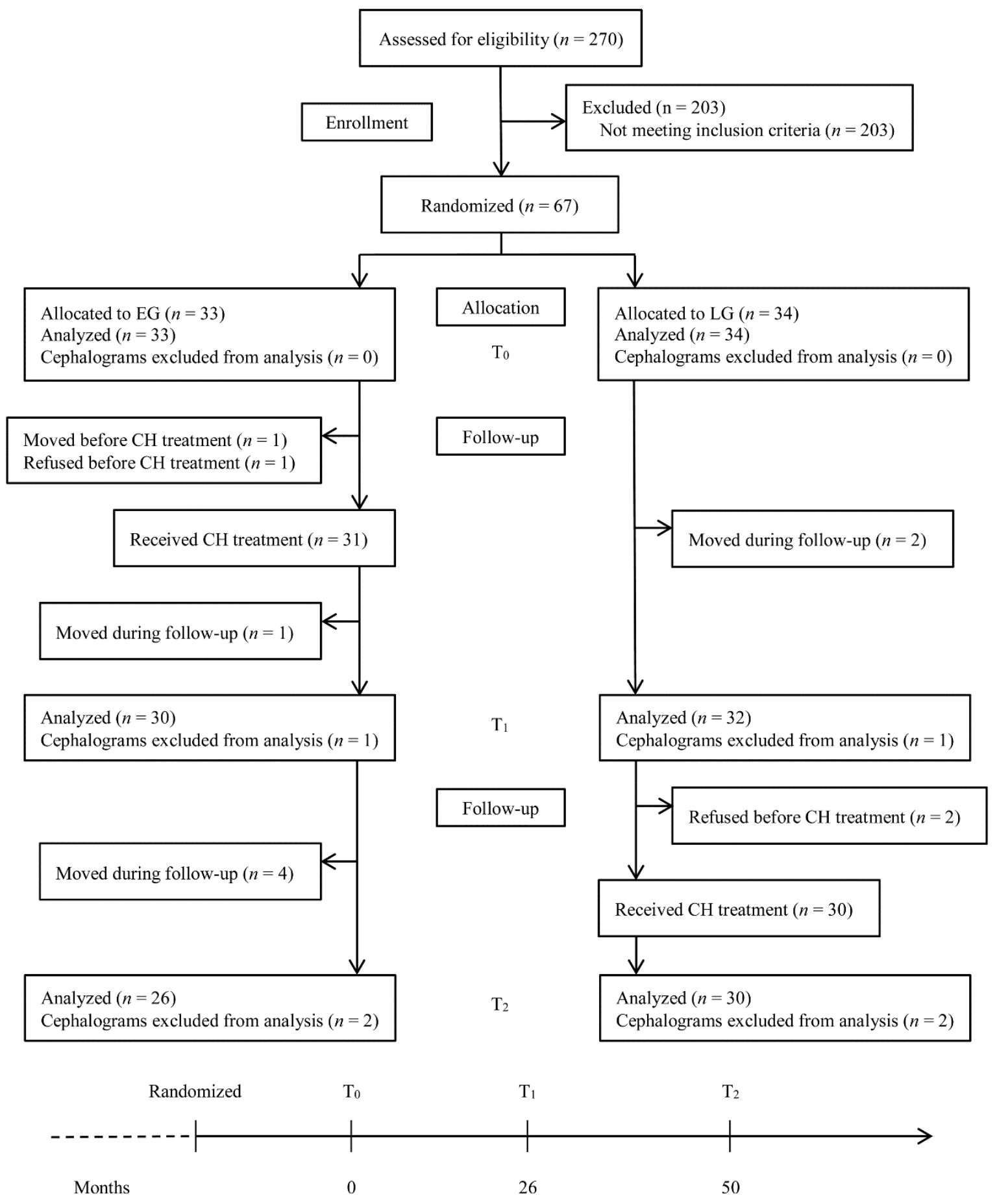

Figure 1. The flow diagram and the timeline of the study. 


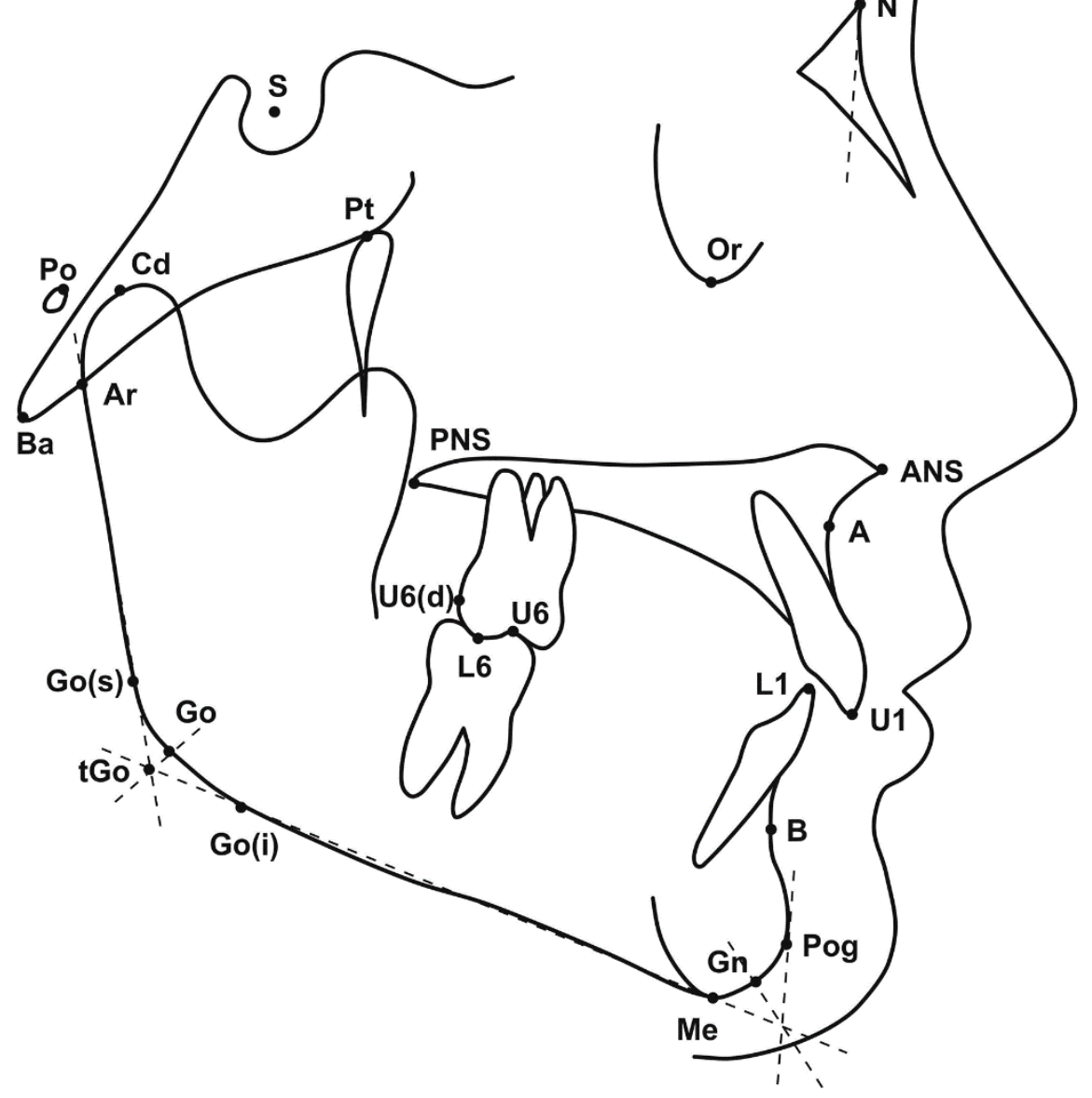

Figure 2. Cephalometric points - S: sella; N: nasion; Pt: the most superior and posterior point of pterygomaxillary fissure; Po: porion; Or; orbitale; Cd: condylion; Ar: articulare; Ba: basion; ANS: anterior nasal spine; PNS: posterior nasal spine; A: subspinale; B: supramentale; Pog: pogonion; Gn: gnathion: a point located on the symphysis constructed by bisecting the angle formed by lines N-Pog and Me-Go(i); Me: menton; Go(s): superior gonion; Go(i): inferior gonion; Go: gonion: a point on the angle of mandible constructed by bisecting the angle formed by lines $\mathrm{Ar}-\mathrm{Go}(\mathrm{s})$ and Me-Go(i); tGo: tangent gonion: the intersection point of lines $\mathrm{Ar}-\mathrm{Go}(\mathrm{s})$ and Me-Go(i); U1: the tip of maxillary central incisor; L1: the tip of mandibular central incisor; U6: the occlusal point of maxillary first molar; L6: the occlusal point of mandibular first molar; U6(d): the most distal point of maxillary first molar. 


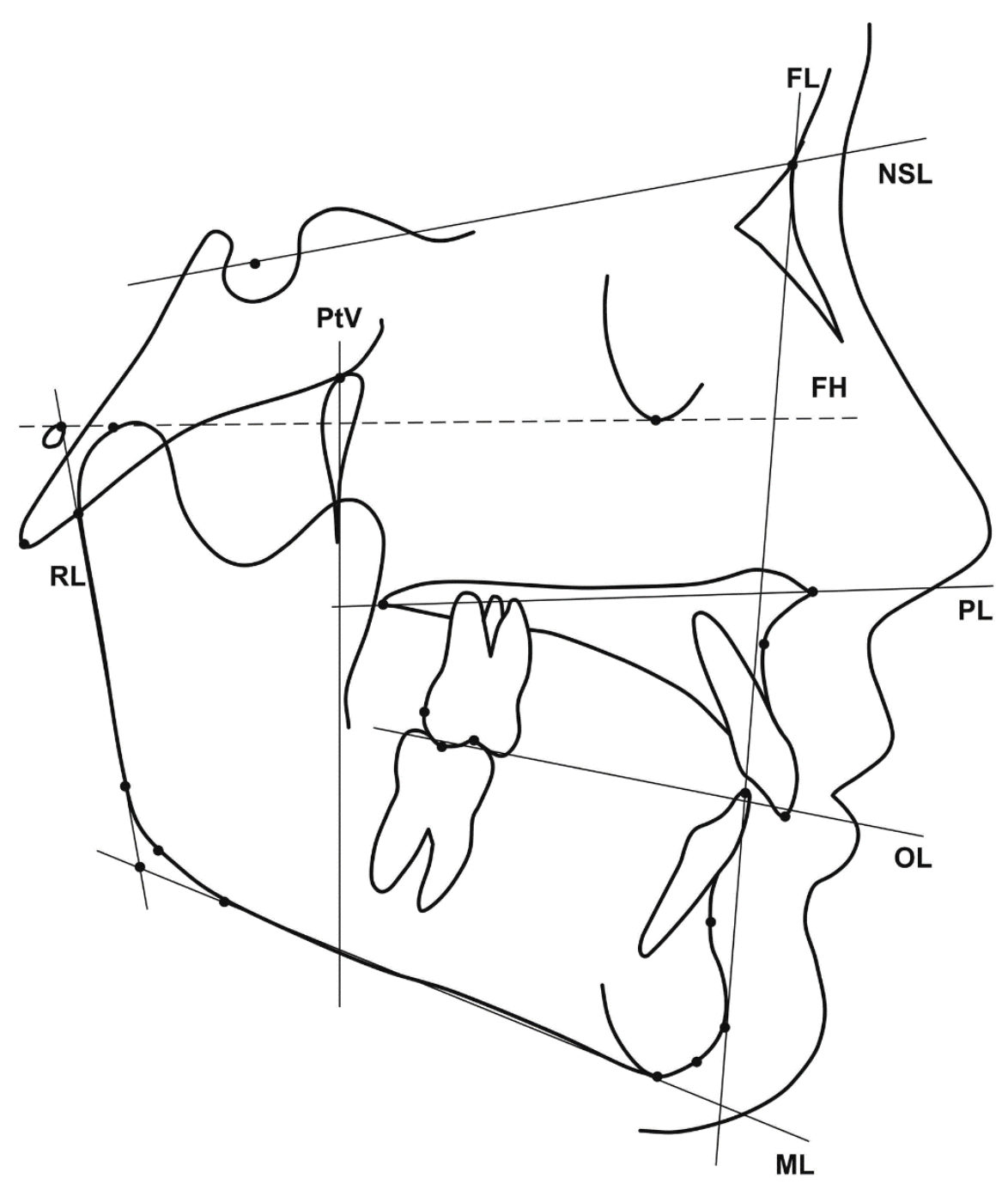

Figure 3. Reference lines - NSL: N-S line; FH: Frankfort horizontal plane: Or-Po line; PL: palatal plane: ANS-PNS line; OL: occlusal plane: the line from the midpoint of U1 and L1 to the midpoint of U6 and L6; ML: mandibular plane: Me-Go(i) line; RL: ramus plane: Ar-Go(s) line; FL: facial plane: N-Pog line; PtV: pterygoid vertical: a line perpendicular to FH from Pt.

Less used cephalometric measurements - U1-FL: the distance of perpendicular line from U1 to facial plane; L1-FL: the distance of perpendicular line from L1 to facial plane; U6(d) -PtV: the distance of perpendicular line from U6(d) to pterygoid vertical; convexity ( $\mathrm{mm}$ ): the distance of perpendicular line from A to facial plane; angle of convexity: the angle formed by intersection of lines N-A and A-Pog; Facial plane angle: the angle of N-S line and facial plane; Gonial angle: the angle formed by ramus plane and mandibular plane; Facial axis: the angle of lines $\mathrm{Ba}-\mathrm{N}$ and $\mathrm{Pt}-\mathrm{Gn}$. 
Table 1. Mean values with 95\% CIs for linear measurements at different time points and change scores during study periods. $P$-value for the group difference.

\begin{tabular}{|c|c|c|c|c|c|c|c|c|c|c|c|c|c|}
\hline & Group & $\mathrm{T}_{0}$ & & \multicolumn{2}{|l|}{$\mathrm{T}_{1}$} & $\mathrm{~T}_{2}$ & \multirow[b]{4}{*}{$95 \%$ CI } & \multirow{2}{*}{$\begin{array}{l}\mathrm{T}_{0}-\mathrm{T}_{1} \\
29\end{array}$} & & \multicolumn{2}{|l|}{$\mathrm{T}_{1}-\mathrm{T}_{2}$} & \multicolumn{2}{|l|}{$\mathrm{T}_{0}-\mathrm{T}_{2}$} \\
\hline & EG $n$ & 33 & & 29 & & 24 & & & & \multicolumn{2}{|l|}{24} & \multicolumn{2}{|l|}{24} \\
\hline & \multirow{2}{*}{ LG $n$} & 34 & & 31 & & 28 & & 31 & & 27 & & 28 & \\
\hline & & Mean & $95 \% \mathrm{CI}$ & Mean & $95 \%$ CI & Mean & & Mean & $95 \% \mathrm{CI}$ & Mean & $95 \%$ CI & Mean & $95 \% \mathrm{CI}$ \\
\hline \multirow[t]{2}{*}{$\mathrm{N}-\mathrm{S}$} & EG & 63.2 & $62.0 ; 64.3$ & 66.3 & $64.3 ; 68.4$ & 65.9 & $64.4 ; 67.4$ & 2.9 & $1.1 ; 4.8$ & -0.2 & $-1.9 ; 1.4$ & 2.8 & $1.3 ; 4.3$ \\
\hline & LG & 63.5 & $61.4 ; 65.6$ & 66.1 & $63.5 ; 68.6$ & 67.6 & $64.9 ; 70.4$ & 2.4 & $0.2 ; 4.5$ & 0.9 & $-2.5 ; 4.2$ & 3.6 & $0.3 ; 7.0$ \\
\hline \multirow[t]{2}{*}{ N-ANS } & EG & 40.1 & $38.7 ; 41.5$ & 45.3 & $45.3 ; 46.5$ & 45.8 & $44.2 ; 47.4$ & $* 5.2$ & $3.3 ; 7.0$ & $* 0.8$ & $-0.2 ; 1.8$ & 5.6 & $4.1 ; 7.1$ \\
\hline & LG & 41.9 & $40.4 ; 43.5$ & 44.3 & $43.3 ; 45.4$ & 47.2 & $46.0 ; 48.4$ & $* 2.4$ & $0.8 ; 4.0$ & $* 2.7$ & $1.5 ; 3.9$ & 5.5 & $3.5 ; 7.4$ \\
\hline \multirow[t]{2}{*}{ ANS-Me } & EG & 55.3 & $53.9 ; 56.6$ & 56.6 & $54.8 ; 58.3$ & 57.0 & $55.7 ; 58.3$ & 1.1 & $-0.6 ; 2.9$ & 0.7 & $-0.8 ; 2.3$ & 1.5 & $-0.3 ; 3.2$ \\
\hline & LG & 55.1 & $52.9 ; 57.3$ & 58.2 & $55.9 ; 60.6$ & 58.1 & $56.2 ; 59.9$ & 2.9 & $0.8 ; 5.0$ & -0.4 & $-2.7 ; 1.8$ & 2.7 & $0.5 ; 4.9$ \\
\hline \multirow[t]{2}{*}{$\mathrm{N}-\mathrm{Me}$} & EG & 93.0 & $91.1 ; 95.0$ & 100.0 & $97.4 ; 102.6$ & 101.1 & $98.6 ; 103.7$ & 6.9 & $4.1 ; 9.7$ & 1.6 & $-0.4 ; 3.7$ & 7.8 & $5.4 ; 10.3$ \\
\hline & LG & 94.9 & $91.5 ; 98.3$ & 100.3 & $97.6 ; 103.1$ & 103.3 & $101.0 ; 105.5$ & 5.1 & $2.8 ; 8.1$ & 2.6 & $-0.3 ; 5.5$ & 8.3 & $4.7 ; 12.0$ \\
\hline \multirow[t]{2}{*}{$\mathrm{Cd}-\mathrm{A}$} & EG & 74.9 & $73.6 ; 76.2$ & 78.4 & $76.0 ; 80.8$ & 79.0 & $76.9 ; 81.1$ & 3.0 & $0.8 ; 5.3$ & 1.1 & $-0.9 ; 3.2$ & 4.1 & $2.1 ; 6.1$ \\
\hline & LG & 76.0 & $73.3 ; 78.8$ & 81.1 & $78.2 ; 84.0$ & 81.1 & $79.3 ; 83.0$ & 4.8 & $2.0 ; 7.6$ & -0.4 & $-3.9 ; 3.1$ & 4.9 & $1.0 ; 8.7$ \\
\hline \multirow[t]{2}{*}{$\mathrm{Cd}-\mathrm{Gn}$} & EG & 91.6 & $90.2 ; 93.0$ & 98.0 & $95.2 ; 100.7$ & 99.1 & $96.8 ; 101.4$ & 6.1 & $3.6 ; 8.6$ & 1.7 & $-0.8 ; 4.3$ & 7.6 & $5.5 ; 10.0$ \\
\hline & LG & 92.7 & $89.1 ; 96.3$ & 100.0 & $96.6 ; 103.4$ & 101.1 & $98.9 ; 103.3$ & 6.7 & $3.3 ; 10.1$ & 0.7 & $-3.4 ; 4.9$ & 8.3 & $3.9 ; 12.7$ \\
\hline \multirow[t]{2}{*}{ Cd-Go } & EG & 46.1 & $44.8 ; 47.4$ & 49.1 & $47.4 ; 50.8$ & 50.5 & $49.0 ; 52.0$ & 3.0 & $1.3 ; 4.6$ & 1.4 & $-0.2 ; 3.0$ & 4.3 & $2.9 ; 5.7$ \\
\hline & LG & 46.2 & $44.3 ; 48.1$ & 50.0 & $48.1 ; 51.9$ & 50.3 & $48.7 ; 52.0$ & 3.8 & $1.6 ; 6.0$ & 0.2 & $-2.2 ; 2.7$ & 4.4 & $1.7 ; 7.1$ \\
\hline \multirow[t]{2}{*}{ S-tGo } & EG & 61.3 & $59.6 ; 62.9$ & 66.5 & $64.1 ; 68.9$ & 67.6 & $65.8 ; 69.4$ & 5.2 & $3.5 ; 6.9$ & 1.4 & $-0.2 ; 3.0$ & 6.4 & $4.7 ; 8.1$ \\
\hline & LG & 61.9 & $59.7 ; 64.3$ & 66.8 & $64.5 ; 69.2$ & 68.2 & $66.3 ; 70.2$ & 4.7 & $2.4 ; 7.3$ & 1.2 & $-1.5 ; 3.8$ & 6.4 & $3.8 ; 9.0$ \\
\hline \multirow[t]{2}{*}{ U1-FL } & EG & 6.7 & $5.9 ; 7.6$ & 7.3 & $6.5 ; 8.1$ & 7.8 & $6.9 ; 8.6$ & 0.7 & $-0.1 ; 1.4$ & $* 0.5$ & $-0.2 ; 1.2$ & 0.9 & $0.1 ; 1.6$ \\
\hline & LG & 8.3 & $7.1 ; 9.5$ & 9.0 & $7.8 ; 10.2$ & 8.4 & $7.2 ; 9.6$ & 0.9 & $0.2 ; 1.6$ & $*-0.7$ & $-1.5 ; 0.1$ & 0.3 & $-0.6 ; 1.1$ \\
\hline \multirow[t]{2}{*}{ L1-FL } & EG & 2.7 & $2.0 ; 3.4$ & 2.4 & $1.7 ; 3.2$ & 3.0 & $2.2 ; 3.8$ & -0.2 & $-0.7 ; 0.2$ & 0.7 & $0.3 ; 1.1$ & 0.1 & $-0.2 ; 0.5$ \\
\hline & LG & 3.4 & $2.4 ; 4.3$ & 3.4 & $2.4 ; 4.4$ & 3.7 & $2.6 ; 4.7$ & 0.3 & $-0.1 ; 0.7$ & 0.0 & $-0.6 ; 0.6$ & 0.4 & $-0.4 ; 1.3$ \\
\hline \multirow[t]{2}{*}{$\mathrm{U} 6(\mathrm{~d})-\mathrm{PtV}$} & EG & 9.9 & $8.9 ; 10.9$ & $* * 9.6$ & $8.5 ; 10.7$ & 10.9 & $9.0 ; 12.5$ & $* *-0.6$ & $-1.4 ; 0.2$ & 1.5 & $0.2 ; 2.9$ & 1.1 & $-0.3 ; 2.5$ \\
\hline & LG & 10.5 & $9.7 ; 11.4$ & $* * 12.0$ & $10.9 ; 13.0$ & 12.4 & $11.2 ; 13.6$ & $* * 1.4$ & $0.6 ; 2.2$ & 0.3 & $-1.2 ; 1.8$ & 1.9 & $0.8 ; 3.1$ \\
\hline \multirow[t]{2}{*}{ convexity (mm) } & EG & 4.0 & $3.2 ; 4.7$ & $* * 2.3$ & $1.6 ; 3.1$ & 2.6 & $1.9 ; 3.2$ & $* * *-1.5$ & $-2.0 ;-1.0$ & $* * 0.2$ & $-0.2 ; 0.6$ & -1.4 & $-1.9 ;-0.8$ \\
\hline & LG & 4.1 & $3.4 ; 4.8$ & $* * 3.8$ & $3.2 ; 4.4$ & 3.1 & $2.2 ; 3.9$ & $* * *-0.1$ & $-0.6 ; 0.4$ & $* *-0.7$ & $-1.2 ;-0.3$ & -1.0 & $-1.6 ;-0.4$ \\
\hline
\end{tabular}


Table 2. Mean values with 95\% CIs for angular measurements at different time points and change scores during study periods. $P$-value for the group difference.

\begin{tabular}{|c|c|c|c|c|c|c|c|c|c|c|c|c|c|}
\hline & Group & \multicolumn{2}{|l|}{$\mathrm{T}_{0}$} & \multicolumn{2}{|l|}{$\mathrm{T}_{1}$} & $\mathrm{~T}_{2}$ & \multirow[b]{4}{*}{$95 \% \mathrm{CI}$} & \multirow{2}{*}{$\frac{T_{0}-T_{1}}{29}$} & \multirow[b]{4}{*}{$95 \%$ CI } & \multicolumn{2}{|l|}{$\mathrm{T}_{1}-\mathrm{T}_{2}$} & \multicolumn{2}{|l|}{$\mathrm{T}_{0}-\mathrm{T}_{2}$} \\
\hline & EG $n$ & 33 & & 29 & & 24 & & & & \multicolumn{2}{|l|}{24} & \multicolumn{2}{|l|}{24} \\
\hline & LG $n$ & 34 & & 31 & & 28 & & 31 & & 27 & & 28 & \\
\hline & & Mean & $95 \% \mathrm{CI}$ & Mean & $95 \% \mathrm{CI}$ & Mean & & Mean & & Mean & $95 \%$ CI & Mean & $95 \% \mathrm{CI}$ \\
\hline \multirow[t]{2}{*}{ SNA } & EG & 82.2 & $80.8 ; 83.7$ & $* * 80.2$ & $78.7 ; 81.6$ & 80.8 & $79.0 ; 82.6$ & $* * *-2.1$ & $-2.8 ;-1.4$ & $* * 0.6$ & $-0.3 ; 1.5$ & -1.5 & $-2.2 ;-0.8$ \\
\hline & LG & 82.4 & $81.1 ; 83.6$ & $* * 83.2$ & $81.8 ; 84.5$ & 81.7 & $80.5 ; 83.0$ & $* * * 1.0$ & $-0.1 ; 2.1$ & $* *-1.4$ & $-2.4 ;-0.5$ & -0.7 & $-1.6 ; 0.3$ \\
\hline \multirow[t]{2}{*}{ SNB } & EG & 77.2 & $76.0 ; 78.5$ & 76.8 & $75.5 ; 78.2$ & 77.3 & $75.5 ; 79.1$ & $*-0.4$ & $-1.0 ; 0.2$ & 0.4 & $-0.3 ; 1.0$ & 0.2 & $-0.5 ; 0.9$ \\
\hline & LG & 77.2 & $76.1 ; 78.3$ & 78.3 & $77.1 ; 79.4$ & 77.9 & $76.8 ; 78.9$ & $* 1.1$ & $0.0 ; 2.2$ & -0.5 & $-1.4 ; 0.3$ & 0.7 & $-0.2 ; 1.5$ \\
\hline \multirow[t]{2}{*}{ ANB } & EG & 5.0 & $4.2 ; 5.7$ & $* * 3.3$ & $2.6 ; 4.1$ & 3.5 & $2.9 ; 4.2$ & $* * *-1.8$ & $-2.4 ;-1.1$ & $* * * 0.3$ & $-0.2 ; 0.7$ & -1.6 & $-2.2 ;-1.1$ \\
\hline & LG & 5.2 & $4.5 ; 5.8$ & $* * 4.9$ & $4.3 ; 5.5$ & 3.9 & $3.2 ; 4.6$ & $* * *-0.1$ & $-0.7 ; 0.5$ & $* * *-0.9$ & $-1.3 ;-0.5$ & -1.3 & $-1.9 ;-0.7$ \\
\hline \multirow[t]{2}{*}{ angle of convexity } & EG & 10.6 & $8.8 ; 12.4$ & $* 6.8$ & $5.0 ; 8.6$ & 7.2 & $5.7 ; 8.7$ & $* * *-4.0$ & $-5.5 ;-2.6$ & $* * 0.5$ & $-0.5 ; 1.5$ & -3.7 & $-5.3 ;-2.6$ \\
\hline & LG & 11.2 & $9.5 ; 13.0$ & $* 10.1$ & $8.6 ; 11.6$ & 8.1 & $6.3 ; 10.0$ & $* * *-0.5$ & $-1.7 ; 0.7$ & $* *-1.9$ & $-2.9 ; 1.0$ & -3.0 & $-4.3 ;-1.8$ \\
\hline \multirow[t]{2}{*}{ Facial plane angle } & EG & 76.8 & $75.6 ; 78.0$ & 76.8 & $75.6 ; 78.0$ & 77.2 & $75.6 ; 78.9$ & 0.1 & $-0.6 ; 0.7$ & 0.4 & $-0.3 ; 1.1$ & 0.7 & $-0.1 ; 1.5$ \\
\hline & LG & 76.6 & $75.4 ; 77.7$ & 78.1 & $76.8 ; 79.4$ & 77.7 & $76.5 ; 78.9$ & 1.3 & $0.2 ; 2.4$ & -0.4 & $-1.4 ; 0.5$ & 1.0 & $0.2 ; 1.8$ \\
\hline \multirow[t]{2}{*}{ NSL-PL } & EG & 3.9 & $2.5 ; 5.4$ & $* 6.4$ & $5.4 ; 7.5$ & 6.0 & $4.7 ; 7.2$ & $* * 2.4$ & $1.0 ; 3.8$ & $* *-0.5$ & $-1.4 ; 0.5$ & 1.9 & $0.4 ; 3.5$ \\
\hline & LG & 5.3 & $4.2 ; 6.3$ & $* 4.8$ & $3.6 ; 5.9$ & 6.6 & $5.7 ; 7.6$ & $* *-0.5$ & $-1.9 ; 0.9$ & $* * 1.9$ & $0.8 ; 3.0$ & 1.7 & $0.6 ; 2.8$ \\
\hline \multirow[t]{2}{*}{ NSL-ML } & EG & 31.7 & $29.8 ; 33.6$ & 31.1 & $29.6 ; 32.6$ & 30.6 & $28.7 ; 32.5$ & -0.6 & $-1.9 ; 0.7$ & -0.3 & $-1.2 ; 0.6$ & -1.5 & $-2.7 ;-0.2$ \\
\hline & LG & 32.5 & $30.8 ; 34.2$ & 31.3 & $29.2 ; 33.3$ & 31.9 & $30.0 ; 33.8$ & -1.2 & $-2.6 ; 0.2$ & 0.6 & $-0.6 ; 1.7$ & -0.7 & $-1.7 ; 0.4$ \\
\hline \multirow[t]{2}{*}{ Gonial angle } & EG & 127.9 & $125.8 ; 129.9$ & 124.6 & $122.8 ; 126.4$ & 123.3 & $121.0 ; 125.5$ & $*-3.4$ & $-4.8 ;-2.0$ & -0.9 & $-2.0 ;-0.3$ & $* *-4.7$ & $-6.2 ;-3.3$ \\
\hline & LG & 127.9 & $125.7 ; 130.1$ & 127.2 & $124.8 ; 129.5$ & 126.3 & $124.0 ; 128.6$ & $*-1.3$ & $-2.4 ;-0.1$ & -1.3 & $-2.6 ;-0.1$ & $* *-2.3$ & $-3.3 ;-1.2$ \\
\hline \multirow[t]{2}{*}{ NSL-RL } & EG & 83.9 & $81.9 ; 85.9$ & 86.5 & $84.6 ; 88.3$ & 87.4 & $85.0 ; 89.8$ & $* 2.8$ & $1.1 ; 4.5$ & 0.6 & $-1.0 ; 2.1$ & 3.2 & $1.8 ; 4.7$ \\
\hline & LG & 84.6 & $82.3 ; 87.0$ & 84.1 & $81.9 ; 86.3$ & 85.6 & $83.7 ; 87.6$ & $* 0.1$ & $-1.8 ; 2.0$ & 1.9 & $0.3 ; 3.5$ & 1.6 & $0.0 ; 3.2$ \\
\hline \multirow[t]{2}{*}{ NSL-OL } & EG & 17.9 & $16.1 ; 19.6$ & 17.1 & $15.6 ; 18.6$ & 17.2 & $15.4 ; 18.9$ & -0.8 & $-2.1 ; 0.5$ & 0.3 & $-0.9 ; 1.5$ & -1.0 & $-2.7 ; 0.7$ \\
\hline & LG & 18.6 & $17.2 ; 20.0$ & 17.0 & $15.3 ; 18.6$ & 16.5 & $15.1 ; 17.8$ & -1.3 & $-2.8 ; 0.1$ & -0.7 & $-2.0 ; 0.6$ & -1.8 & $-2.9 ;-0.8$ \\
\hline \multirow[t]{2}{*}{ OL-ML } & EG & 13.8 & $12.4 ; 15.3$ & 14.0 & $12.9 ; 15.0$ & 13.5 & $12.2 ; 14.7$ & 0.2 & $-0.9 ; 1.3$ & $* * *-0.6$ & $-1.4 ; 0.2$ & -0.4 & $-2.3 ; 1.4$ \\
\hline & LG & 13.9 & $12.4 ; 15.4$ & 14.2 & $12.7 ; 15.8$ & 15.5 & $13.9 ; 17.0$ & 0.1 & $-0.9 ; 1.2$ & $* * * 1.2$ & $0.6 ; 1.9$ & 1.2 & $0.4 ; 2.0$ \\
\hline \multirow[t]{2}{*}{ Facial axis } & EG & 89.2 & $87.7 ; 90.8$ & 88.8 & $87.2 ; 90.3$ & 88.3 & $86.6 ; 90.0$ & -0.7 & $-2.1 ; 0.7$ & -0.6 & $-1.8 ; 0.6$ & -0.8 & $-2.0 ; 0.4$ \\
\hline & LG & 88.7 & $87.5 ; 89.9$ & 89.2 & $87.8 ; 90.6$ & 88.5 & $87.4 ; 89.7$ & 0.1 & $-1.0 ; 1.3$ & -0.7 & $-1.8 ; 0.4$ & -0.5 & $-1.5 ; 0.5$ \\
\hline
\end{tabular}


Table 3. Pearson's correlation coefficients of linear measurements changes in $T_{0}-T_{2}$ to change of SNA in $T_{0}-T_{2}$.

\begin{tabular}{|c|c|c|c|c|}
\hline \multirow[b]{3}{*}{ Change in $T_{0}-T_{2}$} & \multicolumn{4}{|c|}{ Change in SNA $\mathrm{T}_{0}-\mathrm{T}_{2}$} \\
\hline & \multicolumn{2}{|c|}{ EG } & \multicolumn{2}{|l|}{ LG } \\
\hline & $\begin{array}{l}\text { Females } \\
n=10\end{array}$ & $\begin{array}{l}\text { Males } \\
n=14\end{array}$ & $\begin{array}{l}\text { Females } \\
n=12\end{array}$ & $\begin{array}{l}\text { Males } \\
n=16\end{array}$ \\
\hline $\mathrm{N}-\mathrm{S}$ & -0.391 & -0.372 & 0.562 & 0.380 \\
\hline $\mathrm{N}-\mathrm{ANS}$ & $*-0.665$ & $*_{-} 0.641$ & 0.391 & -0.388 \\
\hline ANS-Me & 0.580 & -0.244 & $* 0.617$ & 0.306 \\
\hline $\mathrm{N}-\mathrm{Me}$ & -0.472 & $*_{-} 0.547$ & 0.521 & -0.056 \\
\hline $\mathrm{Cd}-\mathrm{A}$ & -0.124 & $*_{-0.597}$ & $* 0.643$ & 0.265 \\
\hline $\mathrm{Cd}-\mathrm{Gn}$ & 0.226 & $*_{-0.570}$ & $* 0.617$ & 0.261 \\
\hline Cd-Go & 0.494 & $*_{-0.581}$ & 0.464 & 0.231 \\
\hline S-tGo & 0.172 & -0.464 & $* 0.580$ & 0.106 \\
\hline U1-FL & -0.016 & 0.027 & 0.372 & 0.162 \\
\hline $\mathrm{L} 1-\mathrm{FL}$ & -0.079 & -0.168 & 0.252 & 0.181 \\
\hline $\mathrm{U} 6(\mathrm{~d})-\mathrm{PtV}$ & $* 0.712$ & -0.032 & -0.449 & -0.204 \\
\hline convexity (mm) & 0.089 & 0.216 & $* 0.679$ & 0.364 \\
\hline
\end{tabular}

Table 4. Pearson's correlation coefficients of angular measurements changes in $T_{0}-T_{2}$ to change of $S_{N A}$ in $T_{0}-T_{2}$.

\begin{tabular}{|c|c|c|c|c|}
\hline \multirow[b]{3}{*}{ Change in $\mathrm{T}_{0}-\mathrm{T}_{2}$} & \multicolumn{4}{|c|}{ Change in SNA $\mathrm{T}_{0}-\mathrm{T}_{2}$} \\
\hline & \multicolumn{2}{|l|}{ EG } & \multicolumn{2}{|l|}{ LG } \\
\hline & $\begin{array}{l}\text { Females } \\
n=10\end{array}$ & $\begin{array}{l}\text { Males } \\
n=14\end{array}$ & $\begin{array}{l}\text { Females } \\
n=12\end{array}$ & $\begin{array}{l}\text { Males } \\
n=16\end{array}$ \\
\hline \multicolumn{5}{|l|}{ SNA } \\
\hline SNB & $* 0.636$ & $* * 0.741$ & $* * * 0.863$ & $* * 0.742$ \\
\hline ANB & 0.379 & 0.325 & $* 0.623$ & 0.448 \\
\hline angle of convexity & 0.091 & 0.316 & $* 0.667$ & 0.333 \\
\hline Facial plane angle & 0.575 & $* * 0.681$ & $* * 0.724$ & $* * * 0.773$ \\
\hline NSL-PL & $*_{-} 0.656$ & -0.278 & -0.421 & $*_{-0.618}$ \\
\hline NSL-ML & -0.612 & -0.112 & $*_{-0.577}$ & $* *_{-} 0.640$ \\
\hline Gonial angle & -0.148 & 0.276 & 0.312 & 0.239 \\
\hline NSL-RL & -0.236 & -0.474 & $*_{-0.636}$ & $*_{-0.540}$ \\
\hline NSL-OL & -0.093 & -0.468 & $*_{-}-0.804$ & $*_{-0.545}$ \\
\hline OL-ML & -0.362 & 0.326 & 0.311 & -0.083 \\
\hline Facial axis & $* 0.757$ & 0.398 & 0.200 & $* 0.621$ \\
\hline
\end{tabular}

$* P<0.05, * * P<0.01, * * * P<0.001$ 
Table 5. Pearson's correlation coefficients of angular measurements changes in $T_{0}-T_{2}$ to change of Facial axis in $T_{0}-T_{2}$.

\begin{tabular}{|c|c|c|c|c|}
\hline \multirow[b]{4}{*}{ Change in $\mathrm{T}_{0}-\mathrm{T}_{2}$} & \multicolumn{4}{|c|}{ Change in Facial axis $\mathrm{T}_{0}-\mathrm{T}_{2}$} \\
\hline & \multicolumn{2}{|c|}{ EG } & \multicolumn{2}{|l|}{ LG } \\
\hline & Females & Males & Females & Males \\
\hline & $n=10$ & $n=14$ & $n=12$ & $n=16$ \\
\hline SNA & $* 0.757$ & 0.398 & 0.200 & $* 0.621$ \\
\hline SNB & $* 0.663$ & $* 0.639$ & 0.505 & $* * * 0.880$ \\
\hline ANB & 0.065 & -0.340 & -0.395 & -0.279 \\
\hline angle of convexity & -0.268 & -0.424 & -0.301 & -0.365 \\
\hline Facial plane angle & $* 0.702$ & $* * 0.738$ & $* 0.609$ & $* * * 0.857$ \\
\hline NSL-PL & $*_{-} 0.668$ & -0.430 & -0.415 & -0.483 \\
\hline NSL-ML & $*_{-} 0.700$ & -0.480 & -0.411 & $* * *_{-} 0.818$ \\
\hline Gonial angle & 0.112 & 0.033 & *0.611 & 0.391 \\
\hline NSL-RL & -0.473 & $*_{-0.576}$ & $* *-0.741$ & $* *_{-} 0.741$ \\
\hline NSL-OL & -0.612 & $* *-0.692$ & -0.178 & $* * *-0.781$ \\
\hline OL-ML & 0.228 & 0.286 & -0.249 & 0.022 \\
\hline Facial axis & & & & \\
\hline
\end{tabular}

